\title{
Monotonicity and Weighted Prenucleoli: A Characterization Without Consistency
}

\author{
Pedro Calleja, ${ }^{a}$ Francesc Llerena, ${ }^{b}$ Peter Sudhölter ${ }^{c}$ \\ a Department of Mathematics for Economics, Finance and Actuarial Sciences, Barcelona Economic Analysis Team (BEAT), Faculty of \\ Economics and Business, University of Barcelona, 08034 Barcelona, Spain; ${ }^{\mathbf{b}}$ Department of Business Management and Research Center on \\ Industrial and Public Economics (CREIP), Faculty of Business and Economics, Rovira i Virgili University, 43204 Reus, Spain; ${ }^{\mathrm{c}}$ Department of \\ Business and Economics, Faculty of Business and Social Sciences, University of Southern Denmark, 5230 Odense, Denmark \\ Contact: calleja@ub.edu, (D) https://orcid.org/0000-0001-6317-0398 (PC); francesc.llerena@urv.cat, \\ (D) https://orcid.org/0000-0002-4898-8404 (FL); psu@sam.sdu.dk, (D) https://orcid.org/0000-0002-9928-6672 (PS)
}

Received: October 17, 2018

Revised: June 7, 2019

Accepted: June 15, 2019

Published Online in Articles in Advance:

January 6, 2020

MSC2000 Subject Classification: Primary: $91 \mathrm{~A} 12$

OR/MS Subject Classification: Primary: Games/group decisions/cooperative

https://doi.org/10.1287/moor.2019.1022

Copyright: @ 2020 INFORMS

\begin{abstract}
A solution on a set of transferable utility (TU) games satisfies strong aggregate monotonicity (SAM) if every player can improve when the grand coalition becomes richer. It satisfies equal surplus division (ESD) if the solution allows the players to improve equally. We show that the set of weight systems generating weighted prenucleoli that satisfy SAM is open, which implies that for weight systems close enough to any regular system, the weighted prenucleolus satisfies SAM. We also provide a necessary condition for SAM for symmetrically weighted nucleoli. Moreover, we show that the per capita nucleolus on balanced games is characterized by single-valuedness (SIVA), translation covariance (TCOV) and scale covariance (SCOV), and equal adjusted surplus division (EASD), a property that is comparable to but stronger than ESD. These properties together with ESD characterize the per capita prenucleolus on larger sets of TU games. EASD and ESD can be transformed to independence of (adjusted) proportional shifting, and these properties may be generalized for arbitrary weight systems $p$ to I(A)S $S_{p}$. We show that the $p$-weighted prenucleolus on the set

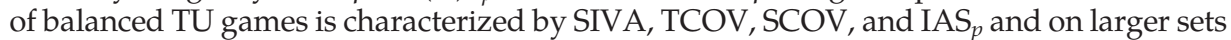
by additionally requiring $\mathrm{IS}_{p}$.
\end{abstract}

Funding: P. Calleja and F. Llerena were supported by Spanish Ministerio de Economía y Competitividad [Grants ECO2016-75410-P and ECO2017-86481-P (AEI/FEDER)]. P. Sudhölter was supported by the Department of Applied Economics IV of the University of the Basque Country at Bilbao and the Spanish Ministerio de Economía y Competitividad [Grant ECO2015-66803-P (MINECO/FEDER)].

Keywords: TU games • weighted prenucleolus $•$ aggregate monotonicity $•$ equal surplus division

\section{Introduction}

The core is one of the most important reference solutions for cooperative games. When restricting the attention to transferable utility (TU) games, there are widely accepted nonempty solutions such as the prenucleolus, a single-valued solution that is a core selection, that is, selects an element of the core whenever the core is nonempty. As a solution in its own right, the prenucleolus may be justified by simple and intuitive axioms. Indeed, in his seminal work, Sobolev [24] proved that the prenucleolus on the set of all TU games with player sets contained in an infinite universe is characterized by single-valuedness (SIVA), anonymity, translation covariance (TCOV), scale covariance (SCOV), and the reduced game property with respect to (w.r.t.) the Davis and Maschler [3] reduced game. It was shown that the equal treatment property replaces anonymity (Orshan [14]) and that if the equal treatment property is used, then SIVA and the reduced game property may be replaced by nonemptiness and the reconfirmation property (Orshan and Sudhölter [15]). However, the infinity assumption of the potential universe of players is crucial in any of the aforementioned axiomatizations of the prenucleolus. It was also shown that it is impossible to suitably modify Peleg's [17] axiomatization of the prekernel that works for a finite universe of players by replacing the converse reduced game property by some minimality principle (see Orshan and Sudhölter [15, corollary 3.8]). To our knowledge, there is only one characterization of the (pre) nucleolus in the literature that does not need varying player sets and consistency. Indeed, Oswald et al. [16] show that the (pre)nucleolus can be characterized with the help of an independence property and continuity. For a detailed comparison with the present characterization, we refer to Remark 5.

Our characterizations do not employ continuity, and they also allow us to characterize weighted prenucleoli rather than just the traditional ones. For symmetrically weighted prenucleoli, there are axiomatizations that are similar to Sobolev's [24] axiomatization of the prenucleolus-only the Davis-Maschler reduced game 
has to be replaced by a suitable new reduced game, the definition of which depends on the weight system (Kleppe et al. [10]). Hence, in this article, we present axiomatizations of the weighted prenucleoli that avoid references to any kind of reduced game and, instead, make use of the fact that all aforementioned weighted prenucleoli are core selections.

Moreover, in order to treat TU games that have an empty core (are not balanced), we employ some kind of monotonicity property in addition. Well-known monotonicity properties that are also satisfied by the core are aggregate monotonicity (AM), strong aggregate monotonicity (SAM), and equal surplus division (ESD). A solution satisfies AM if there is an element according to which nobody is worse off compared with any proposal of the solution of the original TU game if only the worth of the grand coalition is increased. It satisfies ESD if the additional worth of the grand coalition may be distributed equally among the players. For the formal definitions of these intuitive properties, see Section 2. It is well known (see, e.g., Calleja and Llerena [2]) that the per capita prenucleolus satisfies ESD (and, hence, SAM and AM). However, when considering the class of balanced games, ESD is a rather weak property. Indeed, when diminishing the worth of the grand coalition, a balanced game may become nonbalanced. Therefore, an arbitrary balanced game may never arise from another balanced game by just increasing the worth of the grand coalition. In order to receive a stronger property that is similar to ESD, satisfied by the core as well, and applicable to balanced games that become unbalanced when exclusively diminishing the worth of the grand coalition, we introduce equal adjusted surplus division (EASD). To this end, we say that a coalition is fully exact (called "tight" by Oswald et al. [16, definition 1]) if each core element assigns to this coalition precisely its worth in the game. Now a solution satisfies EASD if, whenever the worth of any fully exact coalition is diminished proportionally so that the new game remains balanced, adding equal shares to any element of the solution of the new game yields an element of the solution of the original game [see (5) and (6) for the formal definition].

We show that the per capita nucleolus on the set of balanced games with coinciding player sets $N$ of $n \geqslant 2$ elements is axiomatized by SIVA, TCOV, SCOV, and EASD. On all games with player sets N, ESD is needed in addition to characterize the per capita prenucleolus.

It turns out that ESD and EASD can be translated to independence of proportional shifting (IPS) and independence of adjusted proportional shifting (IAPS). A game arises from another game by proportional shifting if the worth of any proper coalition is increased proportionally to its size. Now IPS requires that a solution element of the latter game belong to the solution of the proportionally shifted games as well. The game arises by adjusted proportional shifting if only those coalitions that are not fully exact are shifted, and IAPS refers to the corresponding independence axiom. Hence, the per capita (pre)nucleolus on the set of balanced (all) games is axiomatized by SIVA, TCOV, SCOV, and IAPS (and IPS).

The properties IPS and IAPS may be generalized to any weight system $p$. Instead of shifting proportionally, the shifting of a coalition has to be proportional to the inverse weight of this coalition. We prove that each weighted (pre)nucleolus is characterized by SIVA, TCOV, SCOV, and suitably defined independence axiom(s) for the corresponding weight system. Hence, for example, the (pre)nucleolus is axiomatized without any reference to reduced games. It should be noted that all employed axioms except SIVA are also satisfied by the core.

We now briefly review the contents of this paper. Section 2 offers the necessary notation, recalls the relevant definitions of the considered solutions and related concepts, contains a list of properties of solutions, and provides the well-known Kohlberg [11] criterion. In Section 3, we investigate which weighted nucleoli satisfy SAM. We generalize the inequalities characterizing symmetric weighted nucleoli that satisfy SAM in the threeperson case provided by Housman and Clark [8] to the n-person case. However, the conditions remain necessary only in the general $n$-person case. Without assuming symmetry, we show that a weighted (pre) nucleolus satisfies regular SAM as defined by Calleja and Llerena [2] if and only if the weight system is regular (i.e., associated with a positive payoff vector). As a consequence, a weighted prenucleolus satisfies ESD if and only if it is the per capita prenucleolus. We prove the continuity of the mapping that assigns, to each weight system and game, the corresponding weighted prenucleolus and use this continuity to show that the set of weight systems generating weighted prenucleoli that satisfy SAM is open, implying, in particular, that SAM is satisfied by any weighted prenucleolus if the weights are close enough to some regular weight system. Section 4 is devoted to the axiomatization of the per capita (pre)nucleolus without making use of any reduced game property. Section 5 presents the new properties IPS and IAPS; shows that these properties are equivalent to ESD and EASD, respectively, for solutions that satisfy TCOV; and that TCOV is crucial. In Section 6, IPS and IAPS are generalized to IS $p$ and IAS $p$, the corresponding properties depending on the weight system $p$. Thus, the $p$-weighted nucleolus on the set of balanced games is characterized by SIVA, TCOV, SCOV, and IAS, whereas $\mathrm{IS}_{p}$ is needed in addition to characterize the $p$-weighted prenucleolus on the unrestricted set of TU games on $N$. Hence, Theorem 4 applied to a weight system $p$ assigning the same weight to any coalition 
provides an axiomatization of the traditional (pre)nucleolus for a fixed set of $n$ players. Moreover, it should be highlighted that nonsymmetrically weighted (pre)nucleoli have not been characterized before. Finally, Section 7 offers some expansions, remarks, and comments.

\section{Notation, Definitions, and Preliminaries}

Let $N$ be a finite nonempty set with $n=|N| \geqslant 2$. A transferable utility game (with player set $N$ ) is a mapping $v: 2^{N} \rightarrow \mathbb{R}$ satisfying $v(\emptyset)=0$. The set of coalitions (nonempty subsets of $N$ ) is denoted by $\mathscr{F}\left(\right.$ i.e., $\mathscr{F}=2^{N} \backslash\{\emptyset\}$ ). We often need the set $\mathscr{\mathscr { F }}:=\mathscr{F} \backslash\{N\}$ of proper coalitions, and the set of all games is denoted by $\Gamma$. Any $x \in \mathbb{R}^{N}$ defines the inessential game $x(\cdot) \in \Gamma$ defined by $x(S)=\sum_{i \in S} x_{i}$ for all $S \in \mathscr{F}$ (and $x(\emptyset)=0$ ). For $v \in \Gamma$, define

$$
\begin{aligned}
X^{*}(v) & =\left\{x \in \mathbb{R}^{N} \mid x(N) \leqslant v(N)\right\}, \text { the set of feasible allocations (payoff vectors), } \\
X(v) & =\left\{x \in \mathbb{R}^{N} \mid x(N)=v(N)\right\}, \text { the set of preimputations, and } \\
C(v) & =\left\{x \in X^{*}(v) \mid x(S) \geqslant v(S) \forall S \in \mathscr{F}\right\}, \text { the core. }
\end{aligned}
$$

Recall that $\mathscr{R} \subseteq \mathscr{F}$ is balanced if there exists $\left(\delta_{S}\right)_{S \in \mathscr{B}}$ such that $\delta_{S}>0$ for all $S \in \mathscr{B}$ and $\sum_{S \in \mathscr{B}} \delta_{S} \mathbb{1}^{S}=\mathbb{1}^{N}$, where $\mathbb{1}^{S} \in \mathbb{R}^{N}$ is the indicator vector of $S$ for any $S \subseteq N$. In such a case, $\left(\delta_{S}\right)_{S \in \mathscr{B}}$ is a system of balancing weights for $\Re$. Then $\mathscr{B}$ is a minimal (w.r.t. set inclusion) balanced collection of coalitions if and only if it has a unique collection of balancing weights. In this case, let $\left(\delta_{S}^{\mathscr{B}}\right)_{S \in \mathscr{B}}$ denote the unique system of balancing weights.

Remark 1. Let $v \in \Gamma$. According to the Bondareva-Shapley theorem (Bondareva [1], Shapley [22]), $C(v) \neq \emptyset$ if and only if

$$
v(N) \geqslant \max \left\{\sum_{S \in \mathscr{B}} \delta_{S}^{\mathscr{B}} v(S) \mid \mathscr{B} \subseteq \mathscr{\mathscr { F }} \text { is minimal balanced }\right\}=: \beta(v)
$$

Hence, games that have nonempty cores are called balanced.

Denote by $\Gamma^{b}$ the set of balanced games. A coalition $S \in \mathscr{F}$ is exact (Schmeidler [20], Shapley [23]) at $v \in \Gamma$ if there exists $x \in C(v)$ that is effective for $S$, that is, $x(S)=v(S)$. Moreover, let us call $S$ fully exact if it is exact and all $x \in C(v)$ are effective for $S$. Let $\mathscr{E}(v)$ denote the set of all fully exact coalitions at $v$, that is,

$$
\mathscr{E}(v)=\{S \in \mathscr{F} \mid S \text { is exact at } v \text { and } x(S)=v(S) \text { for all } x \in C(v)\} .
$$

Hence, if $v \in \Gamma^{b}$, then $N \in \mathscr{E}(v)$. Let $\mathscr{E}(v)=\mathscr{E}(v) \backslash\{N\}$. Then, for any $S \in \mathscr{F} \backslash \mathscr{E}(v)$, there exists $x^{S} \in C(v)$ with $x^{S}(S)>v(S)$. Because $C(v)$ is convex, we conclude that there exists $x \in C(v)$ such that $x(S)>v(S)$ for all $S \in \mathscr{F} \backslash \mathscr{E}(v)$. Note that by the mentioned Bondareva-Shapley theorem, the following relations are valid for any $v \in \Gamma:$

$$
\begin{aligned}
& \beta(v)>v(N) \Longleftrightarrow \mathscr{E}(v)=\emptyset \Longleftrightarrow C(v)=\emptyset, \\
& \beta(v)=v(N) \Longleftrightarrow \mathscr{E}(v) \neq \emptyset, \\
& \beta(v)<v(N) \Longleftrightarrow \mathscr{E}(v)=\{N\} .
\end{aligned}
$$

A solution is a mapping $\sigma$ that assigns a subset $\sigma(v)$ of $X^{*}(v)$ to any $v \in \Gamma$. Its restriction to a set $\Gamma^{\prime} \subseteq \Gamma$ is again denoted by $\sigma$. Moreover, a solution on $\Gamma^{\prime}$ is the restriction to $\Gamma^{\prime}$ of some solution.

A solution $\sigma$ on $\Gamma^{\prime} \subseteq \Gamma$ satisfies

- single-valuedness (SIVA) if $|\sigma(v)|=1$ for all $v \in \Gamma^{\prime}$;

- translation covariance (TCOV) if, for all $v, v^{\prime} \in \Gamma^{\prime}$ such that $v^{\prime}-v=x(\cdot)$ for some $x \in \mathbb{R}^{N}$ (i.e., $v-v^{\prime}$ is inessential), it holds that $\sigma\left(v^{\prime}\right)-\sigma(v)=\{x\}$, that is, $\sigma\left(v^{\prime}\right)=\sigma(v)+\{x\}$, where $A+B$ denotes the Minkowsky sum whenever $A, B \subseteq \mathbb{R}^{N}$;

- scale covariance (SCOV) if $\sigma(\beta v)=\beta \sigma(v)$, whenever $\beta>0$ and $v, \beta v \in \Gamma^{\prime}$.

We often compare the solutions applied to two games $v$ and $v^{\prime}$ such that $v(S)=v^{\prime}(S)$ for all $S \varsubsetneqq N$. Hence, it is useful to define, for each $v \in \Gamma$ and each $\alpha \in \mathbb{R}$, the game $v^{(\alpha)}$ that arises from $v$ by exclusively diminishing the worth of the grand coalition by $n \alpha$. The game $v^{(\alpha)}$ is called the $\alpha$-diminished game of $v$, and it is formally defined by

$$
v^{(\alpha)}(S)= \begin{cases}v(S), & \text { if } S \in \mathscr{\mathscr { F }}, \\ v(N)-n \alpha, & \text { if } S=N .\end{cases}
$$




\section{A solution $\sigma$ on $\Gamma^{\prime} \subseteq \Gamma$ satisfies}

- strong aggregate monotonicity (SAM) if, for all $x \in \sigma\left(v^{(\alpha)}\right)$, there exists $y \in \sigma(v)$ such that $y \gg x$ (i.e., $y_{i}>x_{i}$ for all $i \in N)$, whenever $\alpha>0$ and $v, v^{(\alpha)} \in \Gamma^{\prime}$;

- equal surplus division (ESD) if $\sigma\left(v^{(\alpha)}\right)+\left\{\alpha \mathbb{1}^{N}\right\} \subseteq \sigma(v)$, whenever $\alpha>0$ and $v, v^{(\alpha)} \in \Gamma^{\prime}$.

SIVA is clearly a desirable property of a normative solution, whereas TCOV and SCOV are widely accepted standard properties, together traditionally called covariance under strategic equivalence. Monotonicity properties such as SAM and ESD have been discussed, for example, by Megiddo [13], who showed that the Davis and Maschler [4] bargaining set does not satisfy AM, defined as SAM except that just $y \geqslant x$ is required.

Note that the solutions $C(\cdot), X(\cdot)$, and $X^{*}(\cdot)$ satisfy all foregoing properties except SIVA (provided that $\Gamma^{\prime}$ is rich enough).

We now recall the definition of a "weighted (pre)nucleolus" (see, e.g., Derks and Haller [5, p. 177] and some relevant results of Kleppe et al. [10]). A weight system is a system $p=\left(p_{S}\right)_{S \in \dot{F}}$ such that $p_{S}>0$ for all $S \in \mathscr{F}$. Let $v \in \Gamma$. The p-weighted prenucleolus of $v$, denoted by $\mathscr{P} \mathcal{N}^{p}(v)$, is the set of preimputations $x$ of $v$ that lexicographically minimize the nonincreasingly ordered vector $\left(p_{S} e(S, x, v)\right)_{S \in \dot{F},}$, where $e(S, x, v)=v(S)-x(S)$ is the excess of $S \in \mathscr{F}$ at $x$ w.r.t. $v$. Formally, with $\theta: \mathbb{R}^{\mathscr{F}} \rightarrow \mathbb{R}^{2^{n}-2}$ defined by $\theta_{t}(x)=\max _{\mathscr{T} \subseteq \mathscr{F},|\mathcal{F}|=t} \min _{T \in \mathscr{T}} x_{T}$ for all $t=1, \ldots, 2^{n}-2$,

$$
\mathscr{P} \mathcal{N}^{p}(v)=\left\{x \in X(v) \mid \theta\left(\left(p_{S} e(S, x, v)\right)_{S \in \mathscr{F}}\right) \leqslant_{l e x} \theta\left(\left(p_{S} e(S, y, v)\right)_{S \in \mathscr{F}}\right) \forall y \in X(v)\right\},
$$

where $\leqslant_{\text {lex }}$ denotes the lexicographical order on $\mathbb{R}^{2^{n}-2}$. Note that the $p$-weighted nucleolus of $v$ is defined similarly. Only $X(v)$ is replaced by the set of imputations, that is, by $\left\{x \in X(v) \mid x_{i} \geqslant v(\{i\}) \forall i \in N\right\}$. It is well known that the $p$-weighted prenucleolus is a singleton that we denote by $v^{p}(v)$. Moreover, the $p$-weighted nucleolus is a singleton whenever $v(N) \geqslant \sum_{i \in N} v(\{i\})$. If $v \in \Gamma^{b}$, then both solutions coincide. The foregoing statements may easily be derived from the results of Justman [9]. Replacing $p$ by $\alpha p$ for some positive $\alpha$ does not change the corresponding weighted prenucleolus, and it can be shown that the opposite is also true: $\mathscr{P} \mathcal{N}^{p}=\mathscr{P} \mathcal{N} \mathcal{N}^{\prime}$ if and only if $p$ and $p^{\prime}$ are proportional. If all weights are identical, the corresponding $p$-weighted (pre)nucleolus (point) is the traditional prenucleolus (point) introduced by Schmeidler [19]. If the $p_{S}$ are proportional to $\frac{1}{\mid S S^{\prime}}$ that is, $p_{S}|S|=p_{T}|T|$ for all $S, T \in \mathscr{F}$, then we omit the upper index $p$ and simply write $\mathscr{P} \mathcal{N}(v)=\{v(v)\}$ to denote the per capita prenucleolus (see, e.g., Grotte [6]). Whether a (pre)imputation of a game coincides with its $p$-weighted (pre)nucleolus point can be checked with a suitable modification of Kohlberg's [11] property I or property II. In order to formulate property II $p$ (i.e., Kohlberg's [11] property II for the $p$-weighted prenucleolus), we denote, for any $\alpha \in \mathbb{R}$ and $x \in X(v)$,

$$
\mathscr{D}^{p}(\alpha, x, v)=\left\{S \in \mathscr{\mathscr { F }}^{\prime} \mid p_{S} e(S, x, v) \geqslant \alpha\right\} .
$$

Then $x$ has property $I I_{p}$ if, for any $\alpha \in \mathbb{R}, \mathscr{D}^{p}(\alpha, x, v)$ is balanced or empty. Now proposition 2.2 of Kleppe et al. [10] implies part (a) of the following remark.

Remark 2. Let $v \in \Gamma, p$ be a weight system, and $x \in X(v)$.

(a) Then $x=v^{p}(v)$ if and only if $x$ has property $\mathrm{II}_{p}$.

(b) Let $x=v^{p}(v)$. If $v$ is balanced, as $x$ minimizes the largest $p$-excess, then $x \in C(v)$ and $x(T)>v(T)$ for all $T \in \mathscr{F} \backslash \mathscr{E}(v)$.

It is well known that the weighted prenucleoli satisfy SIVA, TCOV, and SCOV. The specialty of the per capita prenucleolus is that it also satisfies ESD because for any $v \in \Gamma$, any $\alpha \geqslant 0$, any $x \in \mathbb{R}^{N}$, and any $S \in \mathscr{F}$,

$$
\frac{1}{|S|} e\left(S, x, v^{(\alpha)}\right)=\frac{1}{|S|} e\left(S, x+\alpha \mathbb{1}^{N}, v\right)+\alpha ;
$$

that is, the per capita excesses of $v^{(\alpha)}$ at $x$ and those of $v$ at $x+\alpha \mathbb{1}^{N}$ coincide up to a constant.

\section{On Strong Aggregate Monotonicity and Weighted Prenucleoli}

In this section, we introduce regular weight systems that result in weighted prenucleoli satisfying SAM. We show that the set of weight systems generating weighted prenucleoli that satisfy SAM is open. Hence, a $p$-weighted prenucleolus satisfies SAM if, for example, $p$ is close enough to a regular weight system. Moreover, as a consequence of a more general statement, we show that the per capita prenucleolus is the unique weighted 
prenucleolus that satisfies ESD. We also provide a necessary condition for symmetric weight systems to generate aggregate monotonic weighted prenucleoli. Say that a solution $\sigma$ on $\Gamma^{\prime} \subseteq \Gamma$ satisfies

- regular strong aggregate monotonicity (RSAM) if there exists $z \in \mathbb{R}^{N}$ with $z \gg 0$ and $z(N)=n$ such that $\sigma\left(v^{(\alpha)}\right)+\{\alpha z\} \subseteq \sigma(v)$, whenever $\alpha>0$ and $v, v^{(\alpha)} \in \Gamma^{\prime}$. In this case, we say that $\sigma$ satisfies RSAM w.r.t. $z$.

Note that for single-valued solutions, our version of RSAM coincides with the corresponding property introduced by Calleja and Llerena [2]. The following theorem characterizes the weighted prenucleoli that satisfy RSAM.

Theorem 1. Let $\Gamma^{b} \subseteq \Gamma^{\prime} \subseteq \Gamma$, let $p$ be a weight system, and let $z \in \mathbb{R}^{N}$ satisfy $z(N)=n$ and $z \gg 0$. The solution $\mathscr{P} \mathcal{N}^{p}$ on $\Gamma^{\prime}$ satisfies $R S A M$ w.r.t. $z$ if and only if $z(Q) p_{Q}=z(R) p_{R}$ for all $Q, R \in \mathscr{F}$.

Proof. In order to show the if part, let $\widehat{p}_{Q}=\frac{1}{z(Q)}$ for all $Q \in \stackrel{\mathscr{F}}{\mathscr{F}^{\prime}}, \alpha>0, v, v^{(\alpha)} \in \Gamma^{\prime}$, and $x=v^{\hat{p}}\left(v^{(\alpha)}\right)$. Then

$$
\widehat{p}_{S} e(S, x+\alpha z, v)=\frac{v^{(\alpha)}(S)-x(S)-\alpha z(S)}{z(S)}=\widehat{p}_{S} e\left(S, x, v^{(\alpha)}\right)-\alpha \text { for all } S \in \dot{\mathscr{F}}
$$

and $x+\alpha z(N)=v(N)$ so that $v^{\hat{p}}(v)=x+\alpha z$ by Remark 2 .

In order to show the only if part, let $p$ be a weight system such that there are $Q, R \in \mathscr{\mathscr { F }}$ satisfying $z(Q) p_{Q} \neq z(R) p_{R}$. It remains to show that $\mathscr{P} \mathcal{N}^{p}$ does not satisfy RSAM w.r.t. $z$. Choose $\alpha \geqslant \max \left\{z(T) p_{T} \mid T \in \mathscr{F}\right\}$. We now define a collection $\mathscr{S}$ of coalitions by

$$
\mathscr{S}= \begin{cases}\{Q, R\} \cup\{\{i\} \mid i \in N \backslash(Q \cup R)\}, & \text { if } Q \cap R=\emptyset, \\ \{Q, R,(Q \cup R) \backslash(Q \cap R)\} \cup\{\{i\} \mid i \in N \backslash(Q \cup R)\}, & \text { if } Q \cap R \neq \emptyset .\end{cases}
$$

Note that $\mathscr{Y}$ is minimal balanced (with unique balancing weights $\delta_{Q}=\delta_{R}=\delta_{\{i\}}=1$ for all $i \in N \backslash(Q \cup R)$ in the case $Q \cap R=\emptyset$ and $\delta_{Q}=\delta_{R}=\delta_{(Q \cup R) \backslash(Q \cap R)}=\frac{1}{2}$ and $\delta_{\{i\}}=1$ for all $i \in N \backslash(Q \cup R)$ in the case $\left.Q \cap R \neq \emptyset\right)$.

Define $v \in \Gamma$ by

$$
v(S)=\left\{\begin{aligned}
-\frac{\alpha}{p_{S}}, & \text { if } S \in \mathscr{F} \backslash \mathscr{S}, \\
0, & \text { if } S \in \mathscr{Y}, \\
n, & \text { if } S=N .
\end{aligned}\right.
$$

Moreover, let $v^{\prime}=v^{(1)}$, that is, $v^{\prime}(S)=v(S)$ for all $S \in \mathscr{F}$ and $v^{\prime}(N)=0$. Let $x=0 \in \mathbb{R}^{N}$. Then $p_{S} e\left(S, x, v^{\prime}\right)=0$ for all $S \in \mathscr{Y}$, and $p_{T} e\left(T, x, v^{\prime}\right)=-\alpha$ for all $T \in \mathscr{F} \backslash \mathscr{Y}$. Hence, $v^{\prime}$ and $v$ are balanced. Because $\mathscr{Y}$ is balanced, it is straightforward to deduce from part (a) of Remark 2 that $x=v^{p}\left(v^{\prime}\right)$. Let $y=x+z$. It remains to show that $y \neq v^{p}(v)$. Let $\rho=\max \left\{p_{S} e(S, y, v) \mid S \in \mathscr{F}\right\}$ and $\mathcal{T}=\left\{T \in \mathscr{\mathscr { F }} \mid p_{T} e(T, y, v)=\rho\right\}$. Note that for any $S \in \mathscr{S}, p_{S} e(S, y, v)=$ $-p_{S} z(S) \geqslant-\alpha$. Also, for any $T \in \mathscr{F} \backslash \mathscr{S}, p_{T} e(T, y, v)=-\alpha-p_{T} z(T)<-\alpha$ so that $\mathscr{T} \subseteq \mathscr{T}$. Now $p_{R} z(R) \neq p_{Q} z(Q)$ implies that $\mathscr{T} \varsubsetneqq \mathscr{T}$. Because $\mathscr{T}$ is minimal balanced, $\mathscr{T}=\mathscr{D}^{p}(\rho, y, v)$ is not balanced. Hence, Remark 2 shows that $y \neq v^{p}(v)$ so that $\mathscr{P} \mathcal{N}^{p}$ does not satisfy RSAM w.r.t. $z$.

Applied to $z=\mathbb{1}^{N}$, Theorem 1 yields the following corollary.

Corollary 1. For any $\Gamma^{b} \subseteq \Gamma^{\prime} \subseteq \Gamma$, the per capita prenucleolus is the unique weighted prenucleolus that satisfies ESD.

Note that for the only if part of the Theorem 1 (and for the uniqueness part of the preceding corollary), a sufficiently rich set $\Gamma^{\prime} \subseteq \Gamma$ is needed, whereas the remaining if and uniqueness parts are valid for any $\Gamma^{\prime} \subseteq \Gamma$. Indeed, if no properly diminished game of any member of $\Gamma^{\prime}$ belongs to $\Gamma^{\prime}$, then RSAM w.r.t. any $z$ is vacuously satisfied for any $p$-weighted prenucleolus. In the corresponding indirect proofs, we use certain balanced games to construct the desired contradictions.

For $n=2$, any weighted prenucleolus satisfies SAM. Now we turn to SAM assuming $n>2$. Let $p=\left(p_{S}\right)_{S \in \dot{F}}$ be a weight system. Recall that $p$ is symmetric if $p_{S}$ may depend only on $s=|S|$; that is, we write $p_{S}=p(s)$ for all $S \in \mathscr{F}$ in this case. Note that a $p$-weighted prenucleolus satisfies the equal treatment property if and only if it is symmetric (see Kleppe et al. [10, theorem 3.3]). Therefore, mainly symmetrically weighted prenucleoli were discussed in the literature. Housman and Clark [8, theorem 3] show that for a symmetric weight system $p$ in the case $n=3$, the $p$-weighted prenucleolus satisfies SAM if $p(1)>p(2)$ (and AM if $p(1) \geqslant p(2)$ ). We now show that the opposite is also true.

Proposition 1. Let $p$ be a symmetric weight system, and let $s, t \in\{1, \ldots, n-1\}, s \neq t$. If $s p(s) \leqslant(t-1) p(t)$, then $\mathscr{P}_{\mathcal{N}}{ }^{p}$ does not satisfy $S A M$. If $s p(s)<(t-1) p(t)$, then $\mathscr{P}_{\mathcal{N}}^{p}$ does not satisfy AM.

Proof. We may assume that $t>1$. Let $i_{0} \in N, \mathscr{S}=\left\{S \subseteq N \backslash\left\{i_{0}\right\}|s=| S \mid\right\}$, and $\mathscr{T}=\left\{T \subseteq N\left|i_{0} \in T, t=\right| T \mid\right\}$. Note that $\mathscr{S} \cup \mathcal{T}$ is balanced. Indeed, $|\mathcal{T}|=\left(\begin{array}{c}n-1 \\ t-1\end{array}\right)$, and every $j \in N \backslash\left\{i_{0}\right\}$ is a member of $\left(\begin{array}{c}n-2 \\ t-2\end{array}\right)$ elements of $\mathscr{T}$ and a member of 
$\left(\begin{array}{c}n-2 \\ s-1\end{array}\right)$ elements of $\mathscr{Y}$. Therefore, it is straightforward to show that with $\delta_{S}=\frac{(n-t)(s-1) !(n-s-1) !}{(n-1) !}$ for all $S \in \mathscr{S}$ and $\delta_{T}=$ $\frac{(t-1) !(n-t) !}{(n-1) !}$ for all $T \in \mathscr{T}, \Sigma_{R \in \mathscr{S} \cup \mathcal{T}} \delta_{R} \mathbb{1}^{R}=\mathbb{1}^{N}$. Moreover, $\left\{\mathbb{1}^{R} \mid R \in \mathscr{S} \cup \mathcal{T}\right\}$ spans $\mathbb{R}^{N}$ so that, for any $\mathscr{T} \cup \mathcal{T} \subseteq \mathscr{M} \subseteq \mathscr{F}$, we conclude that $\mathscr{B}$ is balanced (see, e.g., Sudhölter [25, remark 2.7(i)]). Choose $v \in \Gamma$ by $v(R)=0$ for all $R \in \mathscr{S} \cup \mathcal{T}$, $v(N)=n$, and $v(S)=\gamma$ for all other $S \in \mathscr{\mathscr { F }}$, where $\gamma$ is some negative constant. Moreover, let $v^{\prime}=v^{(1)}$; that is, $v^{\prime}(S)=$ $v(S)$ for all $S \in \mathscr{\mathscr { F }}$ and $v^{\prime}(N)=0$. Let $x=0 \in \mathbb{R}^{N}$. By Remark $2, x=v^{p}\left(v^{\prime}\right)$. With $a=\frac{n p(t)}{s p(s)+(n-t) p(t)^{\prime}}$, define $y \in \mathbb{R}^{N}$ by $y_{i}=a$ for all $i \in N \backslash\left\{i_{0}\right\}$ and $y_{i_{0}}=n-(n-1) a$. Then $y \in X(v)$ and $p(|R|) e(R, y, v)=-\frac{s n p(s) p(t)}{s p(s)+(n-t) p(t)}=b$ for all $R \in \mathscr{Y} \cup \mathscr{T}$, and if $\gamma$ is small enough, $p(|Q|) e(Q, y, v)<b$ for all $Q \in \mathscr{F} \backslash(\mathscr{S} \cup \mathscr{T})$. Hence, by Remark $2, y=v^{p}(v)$. Now, if $\mathscr{P} \mathcal{N}^{p}$ satisfies SAM, then $y \gg x$, that is, $n-(n-1) a>0$. Inserting the formula for $a$ and eliminating the denominator yield $n s p(s)+n(n-t) p(t)-n(n-1) p(t)>0$, that is, $s p(s)>(t-1) p(t)$. Similarly, if $\mathscr{P} \mathcal{N}^{p}$ satisfies AM, we receive $s p(s) \geqslant$ $(t-1) p(t)$.

Note that Proposition 1, applied to $s=1$ and $t=3$, reproves that the traditional nucleolus (all weights coincide) does not satisfy aggregate monotonicity if $n \geqslant 4$ (see also Megiddo [13] for $n \geqslant 9$ ), which was already shown by Hokari [7] even for the set of convex games.

As mentioned earlier, the opposite statement of Proposition 1 is correct for $n=3$ (Housman and Clark [8]), but the inequalities $s p(s)>(t-1) p(t)$ for all $s, t \in\{1, \ldots, n-1\}$ do not guarantee that the $p$-weighted prenucleolus satisfies SAM. For instance, it may be shown similarly to Proposition 1 that $2 p(3)>p(2)$ is necessary for SAM if $n>4$.

In general, we do not have a characterization of the weight systems $p$ such that the $p$-weighted prenucleoli satisfy SAM. However, we now show that the set of weight systems $p$, not necessarily symmetric, generating $p$-weighted prenucleoli that satisfy SAM is open.

To this end, the following two lemmas are needed. Let $\mathscr{W} \subseteq \mathbb{R}^{\mathscr{F}}$ denote the set of all weight systems. A suitable adjustment of the well-known proof of the continuity of the classical prenucleolus allows us to show that any weighted prenucleolus is continuous as well. However, we need the following stronger result.

Lemma 1. Let $f: \Gamma \times \mathcal{W} \rightarrow \mathbb{R}^{N}$ defined by $f(v, p)=v^{p}(v)$ for all $v \in \Gamma$ and $p \in \mathcal{W}$. Then $f$ is continuous.

Proof. Let $v, v^{k} \in \Gamma$ and $p, p^{k} \in \mathcal{W}$ for all $k \in \mathbb{N}$ such that $\lim _{k \rightarrow \infty} v^{k}=v$ and $\lim _{k \rightarrow \infty} p^{k}=p$. For each $k \in \mathbb{N}$, let $x^{k}=v^{p^{k}}\left(v^{k}\right)$

Claim 1. The set $\left\{x^{k} \mid k \in \mathbb{N}\right\}$ is bounded. Indeed, let $y \in \mathbb{R}^{N}$ be such that $y(N) \leqslant \inf _{k \in \mathbb{N}} v^{k}(N)$, which is finite because $\left\{v^{k}(N) \mid k \in \mathbb{N}\right\}$ is bounded. Moreover, $\left\{p_{S}^{k} e\left(S, y, v^{k}\right) \mid S \in \mathscr{F}^{\circ}, k \in \mathbb{N}\right\}$ is bounded so that $d=\sup _{k \in \mathbb{N}, S \in \mathscr{F}} p_{S}^{k} e\left(S, y, v^{k}\right)$ exists. Now choose $z^{k} \in \mathbb{R}^{N}$ such that $z^{k} \geqslant y$ and $z^{k}(N)=v^{k}(N)$. Then $p_{S}^{k} e\left(S, y, v^{k}\right) \geqslant p_{S}^{k} e\left(S, z^{k}, v^{k}\right)$ for all $S \in \mathscr{F}^{\circ}$ and $\max _{S \in \mathscr{F}} p_{S}^{k} e\left(S, x^{k}, v^{k}\right) \leqslant \max _{S \in \mathscr{F}} p_{S}^{k} e\left(S, z^{k}, v^{k}\right) \leqslant d$. We conclude that for all $k \in \mathbb{N}$, we have

$$
x^{k}(N)=v^{k}(N) \text { and } p_{S}^{k} e\left(S, x^{k}, v^{k}\right) \leqslant d \text { for all } S \in \check{\mathscr{F}} .
$$

In particular, $p_{\{i\}}^{k}\left(v^{k}(\{i\})-x_{i}^{k}\right) \leqslant d$, that is, $x_{i}^{k} \geqslant v^{k}(\{i\})-d / p_{\{i\}}^{k}$, for all $k \in \mathbb{N}$ and $i \in N$, and our claim follows.

Let $\left(x^{k_{j}}\right)_{j \in \mathbb{N}}$ be a convergent subsequence of $\left(x^{k}\right)_{k \in \mathbb{N}}$, and let $x=\lim _{j \rightarrow \infty} x^{k_{j}}$. We have to prove that $x=v^{p}(v)$. Let $\alpha \in \mathbb{R}$ be such that $\mathscr{D}^{p}(\alpha, x, v) \neq \emptyset$. By Remark 2 , it remains to show that $\mathscr{D}^{p}(\alpha, x, v)$ is balanced. Choose $\varepsilon>0$ such that $\mathscr{D}^{p}(\alpha-2 \varepsilon, x, v)=\mathscr{D}^{p}(\alpha, x, v)$. Because $\lim _{j \rightarrow \infty} x^{k_{j}}=x, \lim _{j \rightarrow \infty} v^{k_{j}}=v$, and $\lim _{j \rightarrow \infty} p^{k_{j}}=p$, there exists $J \in \mathbb{N}$ such that

$$
p_{S} e(S, x, v)-\varepsilon<p_{S}^{k_{j}} e\left(S, x^{k_{j}}, v^{k_{j}}\right)<p_{S} e(S, x, v)+\varepsilon \text { for all } S \in \stackrel{\mathscr{F}}{ } \text { and } j>J .
$$

Therefore, $\mathscr{D}^{p}(\alpha, x, v)=\mathscr{D}^{p^{k_{j}}}\left(\alpha-\varepsilon, x^{k_{j}}, v^{k_{j}}\right)$ for all $j>J$. Because $x^{k_{j}}=v^{p^{k_{j}}}\left(v^{k_{j}}\right)$ for all $j \in \mathbb{N}, \mathscr{D}^{p}(\alpha, x, v)$ is balanced by Remark 2. $\square$

In order to prove the second lemma, it is useful to introduce some notation. An ordered partition of $\mathscr{F}^{\circ}$ is a system $\left(\mathscr{B}_{1}, \ldots, \mathscr{B}_{t}\right)$ for some $t \in \mathbb{N}$ such that for all $j, k \in\{1, \ldots, t\}$ with $j \neq k, \emptyset \neq \mathscr{B}_{k} \subseteq \mathscr{F}_{\text {, }}, \mathscr{B}_{j} \cap \mathscr{B}_{k}=\emptyset$, and $\bigcup_{\ell=1}^{t} \mathscr{B}_{\ell}=\mathscr{\mathscr { F }}$. Let $\mathbb{B}=\left(\mathscr{B}_{1}, \ldots, \mathscr{B}_{t}\right)$ be an ordered partition, $p \in \mathscr{W}$, and $x \in \mathbb{R}^{N}$. For each $j \in\{1, \ldots, t\}$, let $\left(\mathscr{B}_{1}^{j}, \ldots, \mathscr{S}_{t_{j}}^{j}\right)$ be the ordered partition of $\mathscr{B}_{j}$ such that for all $i, \ell \in\left\{1, \ldots, t_{j}\right\}$ with $\ell<t_{j},(\mathrm{a}) \mathscr{B}_{i}^{j} \neq \emptyset$, (b) $p_{S} x(S)=$ $p_{T} x(T)$ for all $S, T \in \mathscr{B}_{i}^{j}$, and (c) $p_{S} x(S)<p_{T} x(T)$ for all $S \in \mathscr{B}_{\ell}^{j}$ and $T \in \mathscr{B}_{\ell+1}^{j}$. We put

$$
\mathbb{B}_{x}^{p}=\left(\mathscr{B}_{1}^{1}, \ldots, \mathscr{S}_{t_{1}}^{1}, \ldots, \mathscr{S}_{1}^{t}, \ldots, \mathscr{B}_{t_{t}}^{t}\right)
$$

and note that $\mathbb{B}_{x}^{p}$ is a refinement of $\mathbb{B}$. 
Moreover, we say that $\mathbb{B}$ is a configuration if $\cup_{\ell=1}^{k} \mathscr{B}_{\ell}$ is balanced for all $k \in\{1, \ldots, t\}$.

Let $v \in \Gamma, p \in W, x \in \mathbb{R}^{N}$, and $\mathbb{B}_{v, x}^{p}=\left(\mathscr{B}_{1}, \ldots, \mathscr{B}_{t}\right)$ be the ordered partition defined by the requirements that for all $j, k \in\{1, \ldots, t\}$ with $j<t$, (a) $\mathscr{B}_{k} \neq \emptyset$, (b) $p_{S} e(S, x, v)=p_{T} e(T, x, v)$ for all $S, T \in \mathscr{B}_{k}$, and (c) $p_{S} e(S, x, v)>p_{T} e(T, x, v)$ for all $S \in \mathscr{B}_{j}$ and $T \in \mathscr{B}_{j+1}$. If $x \in X(v)$, then, by Remark $2, \mathbb{B}_{v, x}^{p}$ is a configuration if and only if $x=v^{p}(v)$.

The following lemma is needed.

Lemma 2. Let $p^{*} \in \mathcal{W}$ be such that the $p^{*}$-weighted prenucleolus satisfies $S A M$. For any configuration $\mathbb{B}$, there exists $\varepsilon>0$ such that for any $p \in \mathbb{W}$ with $\left\|p-p^{*}\right\|<\varepsilon$, where $\|\cdot\|$ denotes the Euclidean norm, there exists $x \in \mathbb{R}^{N}$ such that $x \gg 0$, $x(N)=n$, and $\mathbb{B}_{x}^{p}$ is a configuration.

Proof. We may assume without loss of generality that $p_{S}^{*}<\frac{1}{n}$ for all $S \in \mathscr{\mathscr { F }}$. Let $\mathbb{B}=\left(\mathscr{B}_{1}, \ldots, \mathscr{B}_{t}\right)$ be a configuration and define, for any $p \in \mathscr{W}, v^{p}, w^{p} \in \Gamma$ by $v^{p}(S)=w^{p}(S)=-\frac{j}{p_{S}}$ for all $S \in \mathscr{P}_{j}$ and all $j \in\{1, \ldots, t\}, v^{p}(N)=0$, and $w^{p}(N)=n$. By Remark 2, $v^{p}\left(v^{p}\right)=0 \in \mathbb{R}^{N}$. Moreover, let $x^{p}=v^{p}\left(w^{p}\right)$. Again by Remark 2, $\mathbb{B}_{w^{p}, x^{p}}^{p}$ is a configuration.

Because $v^{p^{*}}$ satisfies SAM, $x^{p^{*}} \gg 0$. By Lemma 1, there exists $\varepsilon>0$ such that $x^{p} \gg 0$ for all $p \in W$ with $\left\|p-p^{*}\right\|<\varepsilon$, and we may assume that $\varepsilon$ is small enough such that, in addition, $p_{S}<\frac{1}{n}$ for all $S \in \mathscr{F}$. We conclude that for all $S \in \mathscr{P}_{j}$ and all $j \in\{1, \ldots, t\},-j>p_{S} e\left(S, x^{p}, w^{p}\right)>-j-1$ because $n=x^{p}(N)>x^{p}(S)>0$ and $p_{S}<\frac{1}{n^{\prime}}$ which guarantees that $\mathbb{B}_{w^{p}, x^{p}}^{p}=\mathbb{B}_{x^{p}}^{p} \quad$ 口

The foregoing lemma enables us to prove the following result.

Theorem 2. The set of weight systems $p$ such that the p-weighted prenucleolus satisfies SAM is open.

Proof. Let $p^{*} \in \mathscr{W}$ be such that the $p^{*}$-weighted prenucleolus satisfies SAM. It remains to show that for any $p \in \mathcal{W}$ close enough to $p^{*}$, the $p$-weighted prenucleolus satisfies SAM as well. By Lemma 2, for any configuration $\mathbb{B}$, there exists $\varepsilon(\mathbb{B})>0$ such that for all $p \in \mathscr{W}$ with $\left\|p-p^{*}\right\|<\varepsilon(\mathbb{B})$, there is $x^{p} \in \mathbb{R}^{N}, x^{p} \gg 0, x^{p}(N)=n$ such that $\mathbb{B}_{x^{p}}^{p}$ is a configuration. Because the number of configurations is finite, there exists $\varepsilon>0$ such that $\varepsilon \leqslant \varepsilon(\mathbb{B})$ for all configurations $\mathbb{B}$. Let $\left\|p-p^{*}\right\|<\varepsilon$. We claim that $\mathscr{P} \mathcal{N}^{p}$ satisfies SAM. Assume, on the contrary, that there exists $v \in \Gamma$ and $\alpha_{0}<0$ such that $v^{p}\left(v^{\left(\alpha_{0}\right)}\right)-v^{p}(v) \gg 0$. By continuity of the $p$-weighted prenucleolus, we may assume that $v^{p}\left(v^{(\alpha)}\right)-v^{p}(v) \ngtr 0$ for all $\alpha$ with $\alpha_{0} \leqslant \alpha<0$. Indeed, if $v^{p}\left(v^{\left(\alpha_{1}\right)}\right) \gg v^{p}(v)$ for some $\alpha_{0}<\alpha_{1}<0$, then $\alpha_{2}=$ $\min \left\{\alpha \in \mathbb{R} \mid \alpha_{0} \leqslant \alpha \leqslant \alpha_{1}, v^{p}\left(v^{(\alpha)}\right) \geqslant v^{p}\left(v^{\left(\alpha_{1}\right)}\right)\right\}$ is well defined by continuity so that we may in this case replace $v$ by $v^{\left(\alpha_{2}\right)}$ and $\alpha_{0}$ by $\alpha_{0}-\alpha_{2}$. Let $x=v^{p}(v)$ and $\mathbb{B}=\mathbb{B}_{v, x}^{p}$. Then there exists $\alpha_{0} \leqslant \delta<0$ such that $\mathbb{B}_{v^{(\delta)}, x-\delta x^{p}}^{p}=\mathbb{B}_{x^{p}}^{p}$ so that, by Remark 2 and Lemma 2, $x-\delta x^{p}=v^{p}\left(v^{(\delta)}\right)$. Because $\delta<0$ and $x^{p} \gg 0, x-\delta x^{p} \gg x$, and the desired contradiction is obtained.

We call a weight system $p$ regular if there exists $z \in \mathbb{R}^{N}$ with $z \gg 0$ and $z(N)=n$ such that $z(Q) p_{Q}=z(R) p_{R}$ for all $Q, R \in \mathscr{F}$. Theorems 1 and 2 have the following immediate consequence.

Corollary 2. For any $p \in \mathcal{W}$ that is close enough to some regular weight system, the p-weighted prenucleolus satisfies SAM.

\section{Characterizations of the Per Capita Prenucleolus}

In order to present our axiomatization of the per capita prenucleolus, first, on the set of balanced games, without employing any reduced game property, we recall that for any $v \in \Gamma^{b}$, by (3), $v^{(\alpha)}$ is balanced if and only if $n \alpha \leqslant v(N)-\beta(v)$. Therefore, if $\beta(v)=v(N)$, there does not exist $\alpha>0$ such that $v^{(\alpha)}$ is still balanced. In order to define a property that is similar to ESD but also applicable when $\beta(v)=v(N)$, we define the following modification of $v^{(\alpha)}$, called the adjusted $\alpha$-diminished game of $v$ and denoted by $v^{(\alpha, a d j)}$. Namely, for $v \in \Gamma^{b}$ and $\alpha \geqslant 0, v^{(\alpha, a d j)} \in \Gamma$ is defined by

$$
v^{(\alpha, a d j)}(S)= \begin{cases}v(S)-\alpha|S|, & \text { if } S \in \mathscr{E}(v), \\ v(S), & \text { if } S \in \mathscr{F} \backslash \mathscr{E}(v) .\end{cases}
$$

Note that if $\alpha>0$ is small enough, then $v^{(\alpha, a d j)}$ remains balanced. Indeed, there exists $x \in C(v)$ such that $x(S)>$ $v(S)$ for all $S \in \mathscr{F} \backslash \mathscr{E}(v)$. Now define $x^{(\alpha)}=x-\alpha \mathbb{1}^{N}$ and observe that $x^{(\alpha)}(T)=v^{(\alpha, a d j)}(T)$ for all $T \in \mathscr{E}(v)$ and $\left|x(S)-x^{(\alpha)}(S)\right|=|S| \alpha<n \alpha$ for $S \in \mathscr{F} \backslash \mathscr{E}(v)$. Hence, $x^{(\alpha)}(S) \geqslant v(S)$ for $\alpha$ small enough.

A solution $\sigma$ on $\Gamma^{\prime} \subseteq \Gamma$ satisfies

- equal adjusted surplus division (EASD) if

$$
\sigma\left(v^{(\alpha, a d j)}\right)+\left\{\alpha \mathbb{1}^{N}\right\} \subseteq \sigma(v),
$$

whenever $\alpha>0$ and $v, v^{(\alpha, a d j)} \in \Gamma^{\prime} \cap \Gamma^{b}$. 
Note that EASD may be interpreted similarly to ESD. Recall that a solution satisfies ESD if the solution to a game contains each proposal that arises from an element of the solution to a game in which just the worth of the grand coalition is diminished, namely, by adding equal shares for each player of this additional worth of the grand coalition. EASD also requires adding equal shares of the additional worth of the grand coalition. This requirement can be justified by observing that the set of fully exact coalitions forms a balanced collection, and thus, with respect to the original game $v$, all players are equally undervalued in the adjusted $\alpha$-diminished game $v^{(\alpha, a d j)}$. Moreover, in order to keep $v^{(\alpha, a d j)}$ balanced, not only is the worth of the grand coalition diminished, but also the worth of any fully exact coalition is diminished proportionally to its size.

Note that $C(\cdot), X(\cdot)$, and $X^{*}(\cdot)$ satisfy EASD. Though EASD of the per capita nucleolus follows from more general results of Sections 5 and 6, we provide a separate argument. If $\alpha>0, v, v^{(\alpha, a d j)} \in \Gamma^{b}, x=v\left(v^{(\alpha, a d j)}\right)$, and $y=x+\alpha \mathbb{1}^{N}$, then $e\left(T, x, v^{(\alpha, a d j)}\right)=e(T, y, v)=0$ for all $T \in \mathscr{E}(v)$ and, for any $S \in \mathscr{F} \backslash \mathscr{E}(v), v^{(\alpha, a d j)}(S)=v^{(\alpha)}(S)$ so that as $x \in C\left(v^{(\alpha, a d j)}\right), e(S, y, v) /|S|=e\left(S, x, v^{(\alpha, a d j)}\right) /|S|-\alpha$ (see (4)), and hence, by Remark 2, $y=v(v)$.

Theorem 3. On $\Gamma^{b}$, the per capita nucleolus is the unique solution that satisfies SIVA, TCOV, SCOV, and EASD.

We have seen that the per capita nucleolus satisfies the properties in Theorem 3, so the existence part is already shown. The uniqueness part of Theorem 3 is implied by Theorem 4(a) applied to a per capita weight system together with Proposition 2. The general proof may be regarded as a generalization of the approach presented in the following illustrating three-person example.

Example 1. Let $N=\{1,2,3\}$ and $v=v_{1}$ be given by

$$
v(\{1\})=3, v(\{2\})=6, v(\{3\})=7, v(\{1,2\})=17, v(\{1,3\})=14, v(\{2,3\})=13, v(N)=32 .
$$

Then $(9,10,13) \in C(v)$, and hence, $\mathscr{E}(v)=\{N\}$; that is, $v^{(\alpha, a d j)}=v^{(\alpha)}$ for all $\alpha>0$. Let $v_{2}=v^{(8 / 3)}$. Then $v_{2}(S)=v(S)$ for all $S \in \mathscr{F}$ and $v_{2}(N)=24$; hence, $(9,8,7) \in C\left(v_{2}\right), v_{2}(\{1,2\})+v_{2}(\{3\})=v_{2}(N)$, and $\mathscr{E}\left(v_{2}\right)=\{N,\{1,2\},\{3\}\}$. Let $v_{3}=v_{2}^{(1, a d j)}$. Hence, $v_{3}(N)=21, v_{3}(\{1,2\})=15, v_{3}(\{3\})=6$, and $C\left(v_{3}\right)=\{(8,7,6)\}$, implying that $\mathscr{E}\left(v_{3}\right)=\mathscr{F} \backslash$ $\{\{1\},\{2\}\}$. With $v_{4}=v_{3}^{(1, a d j)}$, we receive $v_{4}(\{1\})=v(\{1\})=3, C\left(v_{4}\right)=\{(7,6,5)\}$, and $\mathscr{E}\left(v_{4}\right)=\mathscr{F} \backslash\{\{1\}\}$ so that $v_{5}=$ $v_{4}^{(4, a d j)}$ is the additive game defined by the vector $y=(3,2,1)$. Now, if $\sigma$ is a solution that satisfies SIVA, TCOV, and SCOV, then $\sigma\left(v_{5}\right)=\{y\}$, as shown in the proof of Theorem 4(a). If $\sigma$ satisfies EASD in addition, then we receive $y_{4}=y+(4,4,4) \in \sigma\left(v_{4}\right), y_{3}=y_{4}+(1,1,1) \in \sigma\left(v_{3}\right), y_{2}=y_{3}+(1,1,1) \in \sigma\left(v_{2}\right)$, and $x=y_{2}+(8 / 3,8 / 3,8 / 3)=$ $\frac{1}{3}(35,32,29) \in \sigma(N, v)$. Because $\mathscr{E}\left(v_{1}\right) \varsubsetneqq \cdots \varsubsetneqq \mathscr{E}\left(v_{5}\right)$, each of the chosen $\alpha$ values is maximal such that the corresponding $\alpha$-diminished adjusted game is balanced.

In order to reconfirm that, indeed, $x=v(v)$, first notice that possible per capita excesses are $0,-8 / 3,-11 / 3,-14 / 3$, and $-26 / 3$, and they are attained by the coalitions in $\mathscr{E}(v), \mathscr{E}\left(v_{2}\right) \backslash \mathscr{E}(v), \mathscr{E}\left(v_{3}\right) \backslash \mathscr{E}\left(v_{2}\right), \mathscr{E}\left(v_{4}\right) \backslash \mathscr{E}\left(v_{3}\right)$, and $\mathscr{E}\left(v_{5}\right) \backslash \mathscr{E}\left(v_{4}\right)$, respectively. By Remark 2, it remains to check that $\mathscr{E}\left(v_{i}\right) \backslash\{N\}, i=2, \ldots, 5$, is balanced. Now $\mathscr{E}\left(v_{2}\right) \backslash\{N\}$ is a partition and, hence, balanced. Balancing weights of the other collections are, respectively,

$$
\begin{aligned}
& \left(\delta_{\{3\}}, \delta_{\{1,2\}}, \delta_{\{1,3\}}, \delta_{\{2,3\}}\right)=(1 / 3,2 / 3,1 / 3,1 / 3), \\
& \left(\delta_{\{2\}}, \delta_{\{3\}}, \delta_{\{1,2\}}, \delta_{\{1,3\}}, \delta_{\{2,3\}}\right)=(1 / 4,1 / 4,1 / 2,1 / 2,1 / 4), \text { and } \\
& \left(\delta_{\{1\}}, \delta_{\{2\}}, \delta_{\{3\}}, \delta_{\{1,2\}}, \delta_{\{1,3\}}, \delta_{\{2,3\}}\right)=(1 / 3,1 / 3,1 / 3,1 / 3,1 / 3,1 / 3) .
\end{aligned}
$$

Remark 3. (a) Each of the axioms employed in Theorem 3 is logically independent of the remaining axioms. Indeed, $X(\cdot)$, that is, the solution that assigns to each game its set of preimputations, exclusively violates SIVA, and the equal split solution (assigning with each $v \in \Gamma$ the singleton $\{x\}$ defined by $x_{i}=\frac{v(N)}{n}$ ) exclusively violates TCOV. Let $\pi: N \rightarrow\{1, \ldots, n\}$ be an order of $N$, that is, a bijection. For any $v \in \Gamma$, define $a^{\pi}=a^{\pi}(v) \in \mathbb{R}^{N}$ by $a_{i}^{\pi}=v\left(P_{i}^{\pi} \cup\{i\}\right)-$ $v\left(P_{i}^{\pi}\right)$ for all $i \in N$, where $P_{i}^{\pi}=\{j \in N \mid \pi(j)<\pi(i)\}$. Note that the Shapley value (Shapley [21]) of $v$ is the average of all $a^{\pi}(v)$ taken over all orderings of $N$. Now, by its definition, the solution $\left\{a^{\pi}(\cdot)\right\}$ satisfies SIVA, SCOV, and TCOV so that it violates exclusively EASD. Finally, let $z \in \mathbb{R}^{N} \backslash\{0\}$ be such that $z(N) \leqslant 0$ and define $\sigma(v)=\mathscr{P} \mathcal{N}(v)+\{z\}$ for all $v \in \Gamma^{b}$. Then $\sigma$ exclusively violates SCOV.

(b) The foregoing properties of the solutions hold for any $\Gamma^{\prime}$ with $\Gamma^{b} \subseteq \Gamma^{\prime} \subseteq \Gamma$.

(c) However, the solution that assigns the per capita prenucleolus to each game in $\Gamma^{b}$ and $\left\{a^{\pi}(v)\right\}$ to each game in $v \in \Gamma^{\prime} \backslash \Gamma^{b}$ satisfies the four axioms of the theorem because $\left\{a^{\pi}(\cdot)\right\}$ satisfies the former three axioms, $\Gamma^{b}$ is closed under strategic equivalence, and EASD refers only to $\Gamma^{b}$.

Corollary 3. Let $\Gamma^{b} \subseteq \Gamma^{\prime} \subseteq \Gamma$. The per capita prenucleolus on $\Gamma^{\prime}$ is the unique solution that satisfies SIVA, TCOV, SCOV, $E A S D$, and ESD. 
Proof. We have seen that $\mathscr{P} \mathcal{N}$ satisfies ESD, and by Theorem 3, it also satisfies the remaining axioms. For the uniqueness part, let $\sigma$ be a solution that satisfies the axioms. Moreover, let $v \in \Gamma^{\prime}$. If $v$ is balanced, then $\sigma(v)=\mathscr{P} \mathcal{N}(v)$ by Theorem 3. Otherwise, let $\alpha=\frac{v(N)-\beta(v)}{n}$. By SIVA, there exists $x \in \mathbb{R}^{N}$ such that $\sigma(v)=\{x\}$ and, by ESD and SIVA, $\sigma\left(v^{(\alpha)}\right)=\{y\}$, where $y=x-\alpha \mathbb{1}^{N}$. Because $v^{(\alpha)}$ is balanced, $\mathscr{P} \mathcal{N}\left(v^{(\alpha)}\right)=\sigma\left(v^{(\alpha)}\right)$ so that $x=v(v)$ by ESD of $\mathscr{P} \mathcal{N}$.

In view of Remark 3 , each of the axioms employed in Corollary 3 is logically independent of the remaining axioms.

In view of (1), (2), and (3), for solutions on $\Gamma^{b}$, EASD implies ESD. Because both the Shapley value and the per capita prenucleolus satisfy SIVA, TCOV, SCOV, and ESD on $\Gamma^{b}$, it follows that ESD does not replace EASD in Theorem 3 provided that $n \geqslant 3$.

Finally, we note that although ESD is weaker than EASD on $\Gamma^{b}$, it is not satisfied by any weighted nucleolus except the per capita nucleolus by Corollary 1.

\section{Independence of (Adjusted) Proportional Shifting}

Let $v \in \Gamma$ and $\alpha \in \mathbb{R}$. We recall the notation of the " $\alpha$-shift" game $w$ of $v$ (see Sudhölter [25, definition 4.3]):

$$
w(S)= \begin{cases}v(S)+\alpha, & \text { if } S \in \mathscr{\mathscr { F }}, \\ v(S), & \text { if } S=N .\end{cases}
$$

We define the proportional $\alpha$-shift game $v_{(\alpha)}$ of $v$ by

$$
v_{(\alpha)}(S)= \begin{cases}v(S)+\alpha|S|, & \text { if } S \in \check{\mathscr{F}}, \\ v(N), & \text { if } S=N .\end{cases}
$$

Note that

$$
v^{(\alpha)}=v_{(\alpha)}-\alpha \mathbb{1}^{N}(\cdot)
$$

Moreover, if $v \in \Gamma^{b}$, we define the adjusted proportional $\alpha$-shift game of $v$ by

and observe that

$$
v_{(\alpha, a d j)}(S)= \begin{cases}v(S)+\alpha|S|, & \text { if } S \in \mathscr{F} \backslash \mathscr{E}(v), \\ v(S), & \text { if } S \in \mathscr{E}(v)\end{cases}
$$

$$
v^{(\alpha, a d j)}=v_{(\alpha, a d j)}-\alpha \mathbb{1}^{N}(\cdot)
$$

Now we are ready to define our independence axioms. A solution $\sigma$ on $\Gamma^{\prime} \subseteq \Gamma$ satisfies

- independence of proportional shifting (IPS) if $\sigma\left(v_{(\alpha)}\right) \subseteq \sigma(v)$, whenever $\alpha>0$ and $v, v_{(\alpha)} \in \Gamma^{\prime}$;

- independence of adjusted proportional shifting (IAPS) if $\sigma\left(v_{(\alpha, a d j)}\right) \subseteq \sigma(v)$, whenever $\alpha>0$ and $v, v_{(\alpha, a d j)} \in \Gamma^{\prime} \cap \Gamma^{b}$. Hence, by (8) and (10), we have the following result.

Proposition 2. Let $\Gamma^{\prime} \subseteq \Gamma$, and let $\sigma$ be a solution on $\Gamma^{\prime}$ that satisfies TCOV. Then we have the following:

(a) If $\Gamma^{\prime}$ is closed under translations, that is, $v \in \Gamma^{\prime}, x \in \mathbb{R}^{N}$ implies $v+x(\cdot) \in \Gamma^{\prime}$, then $\sigma$ satisfies ESD if and only if $\sigma$ satisfies IPS.

(b) If $\Gamma^{\prime} \cap \Gamma^{b}$ is closed under translations, then $\sigma$ satisfies EASD if and only if $\sigma$ satisfies IAPS.

The following corollary is a special case (that of a per capita weight system) of Theorem 4 of Section 6 . The logical independence of each of the employed axioms is also proved in that section.

Corollary 4. (a) The per capita nucleolus is the unique solution on $\Gamma^{b}$ that satisfies SIVA, TCOV, SCOV, and IAPS.

(b) On an arbitrary $\Gamma^{\prime}, \Gamma^{b} \subseteq \Gamma^{\prime} \subseteq \Gamma$, the per capita prenucleolus is the unique solution that satisfies SIVA, TCOV, SCOV, IAPS, and IPS.

The remainder of this section is devoted to a discussion of the impact of TCOV in Proposition 2.

The solution that assigns to each game $v$ the per capita prenucleolus if $v(N)<0$ and the equal split solution if $v(N) \geqslant 0$ satisfies the two independence axioms but violates each of the equal division axioms, provided that $\Gamma^{\prime} \cap \Gamma^{b}$ is rich enough. Hence, in general, IPS does not imply ESD, nor does IAPS imply EASD.

As mentioned earlier, by the Bondareva-Shapley theorem, there exists a smallest real number $\beta(v)$ such that if $v(N)$ is replaced by $\beta(v)$, the game is balanced. Now we may consider the solution that assigns the equal split solution to any game $v$ satisfying $\beta(v)<0$ and the per capita prenucleolus to any other game. This solution satisfies ESD but violates IPS, provided that $\Gamma^{\prime}$ is rich enough. Hence, in general, ESD does not imply IPS. 
Finally, by induction on

$$
t(v)=\left|\left\{\frac{e(S, x, v)}{|S|} \mid S \in \mathscr{F} \backslash \mathscr{E}(v)\right\}\right|, \text { where } x=v(v),
$$

we define a solution $\sigma$ on $\Gamma^{b}$ that satisfies EASD but violates IAPS: if $t(v)=0$ (i.e., $v$ is inessential), then $\sigma(v)=\mathscr{P} \mathcal{N}(v)$ if $v(N) \leqslant 0$ and $\sigma(v)=X(v)$ if $v(N)>0$. Now, if $t(v) \geqslant 1$, then, with $x=v(v)$, let

$$
\alpha(v)=-\max \left\{\frac{e(S, x, v)}{|S|} \mid S \in \mathscr{F} \backslash \mathscr{E}(v)\right\},
$$

and observe that $\left\{\alpha \geqslant 0 \mid v^{(\alpha, a d j)} \in \Gamma^{b}\right\}=[0, \alpha(v)]$ and $\alpha(v)>0$. Let $v^{\prime}=v^{(\alpha(v), a d j)}$. By EASD of the per capita prenucleolus, $t\left(v^{\prime}\right)=t(v)-1$; hence, $\sigma\left(v^{\prime}\right)$ is already defined by the inductive hypothesis. We now define $\sigma(v)=\sigma\left(v^{\prime}\right)+\left\{\alpha(v) \mathbb{1}^{N}\right\}$. Again, by induction on $t(v)$, it may be shown that $\sigma$ satisfies EASD. However, it does not satisfy IAPS.

Remark 4. We now verify that EASD and SIVA imply IAPS for any solution $\sigma$ on $\Gamma^{b}$. Indeed, let $\sigma$ satisfy SIVA and EASD. Let $v \in \Gamma^{b}, \alpha>0$, and $w=v+\alpha \mathbb{1}^{N}(\cdot)$. By (10), it suffices to prove that $\sigma(w)=\sigma(v)+\left\{\alpha \mathbb{1}^{N}\right\}$. We proceed by induction on $t(v)$ defined by (11). If $t(v)=0$, that is, $v$ is inessential $(\mathscr{E}(v)=\mathscr{F}(v))$, then $w$ is also inessential (i.e., $\mathscr{E}(w)=\mathscr{E}(v))$, so $v=w^{(\alpha, a d j)}$, and the proof is finished by SIVA and EASD. If $t(v)>0$, then TCOV of the per capita nucleolus guarantees that $\mathscr{E}(v)=\mathscr{E}(w)$ and $\alpha(v)=\alpha(w)$, where $\alpha(v)$ and $\alpha(w)$ are defined as in (12), and with $v^{\prime}=v^{(\alpha(v), a d j)}$ and $w^{\prime}=w^{(\alpha(v), a d j)}, t\left(v^{\prime}\right)=t\left(w^{\prime}\right)=t(v)-1$, and $w^{\prime}=v^{\prime}+\alpha \mathbb{1}^{N}(\cdot)$. Hence, by the inductive hypothesis, $\sigma\left(w^{\prime}\right)=\sigma\left(v^{\prime}\right)+\left\{\alpha \mathbb{1}^{N}\right\}$, so again by SIVA and EASD, $\sigma(v)=\sigma(w)$, and the proof is complete.

\section{Generalizing the Independence Axioms to Arbitrary Weight Systems}

Let $p=\left(p_{S}\right)_{S \in \dot{F}}$ be a weight system, let $v \in \Gamma$, and let $\alpha \in \mathbb{R}$. Define the $(\alpha, p)$-shift game $v_{(\alpha, p)} \in \Gamma$ by

$$
v_{(\alpha, p)}(S)= \begin{cases}v(S)+\frac{\alpha}{p_{S}}, & \text { if } S \in \mathscr{\mathscr { F }}, \\ v(N), & \text { if } S=N,\end{cases}
$$

and if $v \in \Gamma^{b}$, define the adjusted $(\alpha, p)$-shift game $v_{(\alpha, p, a d j)} \in \Gamma$ by

$$
v_{(\alpha, p, a d j)}(S)= \begin{cases}v(S)+\frac{\alpha}{p_{S}}, & \text { if } S \in \mathscr{F} \backslash \mathscr{E}(v), \\ v(S), & \text { if } S \in \mathscr{E}(v) .\end{cases}
$$

Now we can suitably modify our independence axioms. A solution $\sigma$ on $\Gamma^{\prime} \subseteq \Gamma$ satisfies

- independence of $p$-shifting $\left(\operatorname{IS}_{p}\right)$ if $\sigma\left(v_{(\alpha, p)}\right) \subseteq \sigma(v)$, whenever $\alpha>0$ and $v, v_{(\alpha, p)} \in \Gamma^{\prime}$;

- independence of adjusted $p$-shifting (IAS $)_{p}$ ) if $\sigma\left(v_{(\alpha, p, a d j)}\right) \subseteq \sigma(v)$, whenever $\alpha>0$ and $v, v_{(\alpha, p, a d j)} \in \Gamma^{\prime} \cap \Gamma^{b}$.

Lemma 3. Let $p$ be a weight system. On any $\Gamma^{\prime} \subseteq \Gamma, \mathscr{P}_{\mathcal{N}}^{p}$ satisfies $I S_{p}$ and $I A S_{p}$.

Proof. Let $v \in \Gamma, x=v^{p}(v), \alpha>0, v^{\prime}=v_{(\alpha, p)}$, and $S \in \mathscr{F}$. Then $p_{S} e\left(S, x, v^{\prime}\right)=p_{S} e(S, x, v)+\alpha$ so that $\mathrm{IS}_{p}$ follows from Remark 2(a). Now assume that $v$ and $w=v_{(\alpha, p, a d j)}$ are balanced. It suffices to show that $v^{p}(w)=x$. Hence, we may assume that $\mathscr{E}(v) \varsubsetneqq \mathscr{F}$. Let $y \in C(w)$. Because $w(N)=v(N)$ and $w(S) \geqslant v(S)$ for all $S \in \mathscr{F}, y \in C(v)$. We conclude that $y(S) \geqslant v(S)+\frac{\alpha}{p_{S^{\prime}}}$, that is, $p_{S} e(S, y, v) \leqslant-\alpha$ for all $S \in \mathscr{F} \backslash \mathscr{E}(v)$. Because $x$ lexicographically minimizes the nonincreasingly ordered vector of $p$-weighted excesses, $p_{S} e(S, x, v) \leqslant-\alpha$ for all $S \in \mathscr{F} \backslash \mathscr{E}(v)$ as well. Hence, $x \in C(w)$ so that $\gamma_{1}=\max \left\{p_{S} e(S, x, v) \mid S \in \mathscr{F} \backslash \mathscr{E}(v)\right\} \leqslant-\alpha$. If $\gamma_{1}<-\alpha$, then

$$
D^{p}(\gamma, x, w)= \begin{cases}D^{p}(\gamma-\alpha, x, v), & \text { if } \gamma<0, \\ D^{p}(0, x, v), & \text { if } \gamma=0, \\ \emptyset, & \text { if } \gamma>0 .\end{cases}
$$

If $\gamma_{1}=-\alpha$, then

$$
D^{p}(\gamma, x, w)= \begin{cases}D^{p}(\gamma-\alpha, x, v), & \text { if } \gamma \leqslant 0 \\ \emptyset, & \text { if } \gamma>0\end{cases}
$$

In any case, $x=v^{p}(w)$ by Remark 2(a). 
Theorem 4. (a) The p-weighted nucleolus is the unique solution on $\Gamma^{b}$ that satisfies SIVA, TCOV, SCOV, and IAS.

(b) On any $\Gamma^{\prime}, \Gamma^{b} \subseteq \Gamma^{\prime} \subseteq \Gamma$, the $p$-weighted prenucleolus is the unique solution that satisfies SIVA, TCOV, SCOV, IAS ${ }_{p}$, and $I S_{p}$.

Proof. (a) It is well known that $\mathscr{P}_{\mathcal{N}^{p}}$ satisfies SIVA, TCOV, and SCOV, and by Lemma 3 , it satisfies IAS . In order to show uniqueness, let $\sigma$ be a solution that satisfies SIVA, TCOV, SCOV, and $\operatorname{IAS}_{p}$. Let $v \in \Gamma^{b}$. By SIVA, $\sigma(v)=\{x\}$ for some $x \in \mathbb{R}^{N}$. Let $y=v^{p}(v)$. It remains to show that $x=y$. Let $t(v)=\left|\left\{p_{S} e(S, y, v) \mid S \in \mathscr{F} \backslash \mathscr{E}(v)\right\}\right|$. We proceed by induction on $t(v)$. If $t(v)=0$, then $v$ is inessential. By TCOV, we may assume that $v(S)=0$ for all $S \subseteq N$. By SIVA and SCOV,$\{\gamma x\}=\sigma(\gamma v)=\sigma(v)$ for all $\gamma>0$. Hence, $x=y=0 \in \mathbb{R}^{N}$. If $t(v)>0$, then let $\alpha=-\max \left\{p_{S} e(S, y, v) \mid S \in\right.$ $\left.\mathscr{F}_{\mathcal{F}} \backslash \mathscr{E}(v)\right\}$. By $\operatorname{IAS}_{p}$ of $\mathscr{P} \mathcal{N}^{p}, y=v^{p}\left(v_{(\alpha, p, a d j)}\right)$ so that $t\left(v_{(\alpha, p, a d j)}\right)=t(v)-1$. By our induction hypothesis, $\sigma\left(v_{(\alpha, p, a d j)}\right)=$ $\{y\}$. Hence, by $\operatorname{IAS}_{p}$ of $\sigma, x=y$.

(b) The $p$-weighted prenucleolus $\mathscr{P} \mathcal{N}^{p}$ satisfies SIVA, TCOV, SCOV, and IAPS $p$ and $\mathrm{IS}_{p}$ by Lemma 3 . To show the uniqueness part, let $\sigma$ be a solution that satisfies the five foregoing axioms. Let $v \in \Gamma^{\prime}$ and $y=v^{p}(v)$. By SIVA, $\sigma(v)=\{x\}$ for some $x \in \mathbb{R}^{N}$, and it remains to show that $x=y$. If $v \in \Gamma^{b}$, then $x=y$ by part (a). Hence, we may assume that $v$ is not balanced. Choose $\alpha \geqslant \max \left\{p_{S} e(S, y, v) \mid S \in \mathscr{\mathscr { F }}\right\}$ and observe that $v^{\prime}=v_{(-\alpha, p)}$ is balanced because $y \in C\left(v^{\prime}\right)$. Because $v=v_{(\alpha, p)}^{\prime}$, by $\mathrm{IS}_{p}$ of $\mathscr{P} \mathcal{N}^{p}, y=v^{p}\left(v^{\prime}\right)$. We have already proved that $\mathscr{P} \mathcal{N}$ coincides with $\sigma$ on balanced games, so $\sigma\left(v^{\prime}\right)=\mathscr{P} \mathcal{N}\left(v^{\prime}\right)=\{y\}$, and the proof is finished by $\mathrm{IS}_{p}$ of $\sigma$.

By means of examples, we now show that each of the properties employed in Theorem 4 is logically independent of the remaining properties. Indeed, $X(\cdot)$ exclusively violates SIVA, the equal split solution exclusively violates TCOV, and the solution that assigns $\mathscr{P} \mathcal{N}^{p}+\{z\}$, where $z \in \mathbb{R}^{N} \backslash\{0\}$ satisfies $z(N) \leqslant 0$, to any game $v$ exclusively violates SCOV. To show that $\mathrm{IAS}_{p}$ is logically independent, let, for any $v \in \Gamma$, $\alpha^{\prime}(v)=\max \left\{\alpha \in \mathbb{R} \mid v_{(\alpha, p)} \in \Gamma^{b}\right\}$, and define $\sigma(v)=\left\{a^{\pi}\left(v_{\left(\alpha^{\prime}(v), p\right)}\right)\right\}$, where $\pi$ is some ordering of $N$ (see Remark 3 for the definition of $\left.a^{\pi}(\cdot)\right)$. Notice that $v_{\left(\alpha^{\prime}\left(v_{(\alpha, p)}\right), p\right)}=v_{\left(\alpha^{\prime}(v), p\right)}$ for all $v \in \Gamma$ and $\alpha \in \mathbb{R}$. Hence, $\sigma$ is well defined. Thus, $\sigma$ satisfies $\mathrm{IS}_{p}$ and SIVA. It is straightforward to check that $\sigma$ also satisfies TCOV and SCOV. Finally, IS $p$ is exclusively violated by the solution that coincides with the $p$-weighted prenucleolus on $\Gamma^{b}$ and with $\left\{a^{\pi}(\cdot)\right\}$ on $\Gamma^{\prime} \backslash \Gamma^{b}$, provided that $\Gamma^{\prime} \backslash \Gamma^{b} \neq \emptyset$, where $\pi$ is some ordering of $N$. To show that there is a solution of this kind that does not coincide with $\mathscr{P}_{\mathcal{N}}^{p}$, choose $v \in \Gamma^{\prime} \backslash \Gamma^{b}$ and $S \in \mathscr{F}^{\circ}$ with $v(S)>x(S)$, where $x=v^{p}(v)$. Choose $i \in S$ and an ordering $\pi$ such that $P_{i}^{\pi}=S \backslash\{i\}$. Hence, with $y=a^{\pi}(v)$, we receive $y(S)=v(S)$; hence, $a^{\pi}(v) \neq v^{p}(v)$.

Remark 5. Let $p$ be a weight system of the traditional prenucleolus, that is, $p_{S}=p_{T}$ for all $S, T \in \mathscr{F}$. It should be noted that in this case, IAS $p$ is related to the property "relative independence of slack coalitions" of Oswald et al. [16, definition 2] that only apparently aims into the opposite direction. In our notation, and generalizing the property suitably to set-valued solutions, a solution $\sigma$ on $\Gamma^{b}$ satisfies relative independence of slack coalitions if $\sigma(w) \subseteq \sigma\left(w_{(-\alpha, p, a d j)}\right)$ for all $w \in \Gamma^{b}$ and all $\alpha>0$. Our IAS $_{p}$ implies relative independence of slack coalitions because with $v=w_{(-\alpha, p, a d j)}, w=v_{(\alpha, p, a d j)}$. However, the mentioned authors need continuity in addition to characterize the traditional nucleolus on $\Gamma^{b}$. The reason is simple. Namely, for the foregoing games $w$ and $v$ compared by relative independence of slack coalitions, $\mathscr{E}(v)=\mathscr{E}(w)$. For $\operatorname{IAS}_{p}$, the situation may differ: if $\mathscr{E}(v) \varsubsetneqq \in \mathscr{F}$, then with $\alpha(v)$ defined by (12), we receive $\mathscr{E}(v) \varsubsetneqq \mathscr{E}\left(v_{(\alpha(v), p, a d j)}\right)$. Therefore, our property is stronger. Indeed, we will now generalize the set-valued version of relative independence of slack coalitions to arbitrary weight systems and provide an example (Example 2) that shows that this property does not replace $\mathrm{IAS}_{p}$ in Theorem 4(a). Hence, as in the characterization of the traditional (pre)nucleolus by Oswald et al. [16], an additional property such as continuity would be needed when replacing IAS $_{p}$ by the aforementioned weaker property.

We say that a solution $\sigma$ on $\Gamma^{b}$ satisfies $p$-relative independence of slack coalitions $(p-R I S C)$ if $\sigma(v) \subseteq \sigma\left(v_{(-\alpha, p, a d j)}\right)$ for all $v \in \Gamma^{b}$ and all $\alpha>0$. The following example shows that $p$-RISC does not replace IAS $p$ in Theorem 4(a), provided that $n \geqslant 3$.

Example 2. Let $n \geqslant 3$ and $k, \ell \in N, k \neq \ell$. Choose $x \in \mathbb{R}^{N}$ with $x(N) \leqslant 0$. For any $t \in \mathbb{R}$, define $w_{t} \in \Gamma^{b}$ by

$$
w_{t}(S)= \begin{cases}-\frac{t}{p_{\{k\}}}, & \text { if } S=\{k\}, \\ -1-\frac{t}{p_{\{\ell\}},} & \text { if } S=\{\ell\}, \\ 0, & \text { if } S \in \mathscr{F} \backslash\{\{k\},\{\ell\}\} .\end{cases}
$$

Note that the core of $w_{t}$ is the singleton $\{0\}$ for any $t \geqslant 0$, and it is empty whenever $t<0$. Moreover, $w_{t}$ is strategically equivalent to $w_{t^{\prime}}$ (i.e., there exists $\beta>0$ and $z \in \mathbb{R}^{N}$ such that $\left.w_{t^{\prime}}=\beta w_{t}+z(\cdot)\right)$ if and only if $t=t^{\prime}$. Hence, we may define our solution $\sigma$ by $\sigma\left(\beta w_{t}+z(\cdot)\right)=\{\beta x+z\}$ for all $\beta, t>0$ and $z \in \mathbb{R}^{N}$ and $\sigma(v)=\left\{v^{p}(v)\right\}$ for all $v \in \Gamma^{b}$ that are not strategically equivalent to any $w_{t}, t>0$. By construction, $\sigma$ satisfies SIVA, SCOV, and 
TCOV. Moreover, if $v \in \Gamma^{b}$ is not strategically equivalent to some $w_{t}$ and $\alpha>0$, then $v_{(-\alpha, p, a d j)}$ is also not strategically equivalent to any $w_{t}$. Finally, with $v=\beta w_{t}+z(\cdot)$ for some $t, \beta>0$ and $z \in \mathbb{R}^{N}$, we receive $v_{(-\alpha, p, a d j)}=\beta w_{t^{\prime}}+z(\cdot)$, where $t^{\prime}=t+\alpha / \beta$. Hence, $\sigma$ satisfies $p$-RISC.

\section{Final Remarks}

First, for any weight system $p$ and any $v \in \Gamma$, the $p$-weighted least core of $v$ is the set

$$
\mathscr{L} C^{p}(v)=\left\{x \in X(v) \mid \max _{S \in \mathscr{F}} p_{S} e(S, x, v) \leqslant \max _{S \in \mathscr{F}} p_{S} e(S, y, v) \text { for all } y \in X(v)\right\} .
$$

Hence, the $p$-weighted least core contains the $p$-weighted (pre)nucleolus. Similarly to Kohlberg's [11] property II for the $p$-weighted prenucleolus (see Remark 2), it may be shown that the $p$-weighted least core is characterized by the following property: let $x \in X(v)$ and $\alpha^{0}(x, v)=\max _{S \in \dot{F}} p_{S} e(S, x, v)$. Then $x \in \mathscr{L} C^{p}(v)$ if and only if $\mathscr{D}^{p}\left(\alpha^{0}(x, v), x, v\right)$ contains a balanced subset. Using this property, it can be shown that $\mathscr{L} C^{p}$ satisfies IAS $_{p}$ and IS $_{p}$ and that the per capita least core satisfies IAPS, IPS, EASD, and ESD. It is well known that $\mathscr{L} C^{p}$ satisfies TCOV and SCOV. Clearly, $\mathscr{L} C^{p}(v)$ is a nonempty and bounded set. Therefore, in our characterization results, SIVA cannot be replaced by nonemptiness (requiring that the solution assigns a nonempty set to any game under consideration) even if Pareto efficiency (requiring that the solution assign a subset of $X(v)$ to each game $v$ under consideration) and boundedness are required in addition.

Second, any $p$-weighted (pre)nucleolus may be regarded as a nucleolus for games with permissible coalitions and permissible preimputations in the sense of Maschler et al. [12, p. 87], who characterize this solution (see their theorem 6.4 and corollary 6.5) on sets of truncated games as the largest solution that satisfies "nondiscrimination" $\left(\mathbf{P}_{1}\right)$, "redundancy" $\left(\mathbf{P}_{2}\right)$, "being contained in the least core" $\left(\mathbf{P}_{3}\right)$, and a "reduced game property" $\left(\mathbf{P}_{13}\right)$ that requires reducing the set of feasible coalitions and the set of preimputaions rather than the player set. Here, truncation requires both allowing restriction of the game to subsets of coalitions and restriction of the set of corresponding preimputations. However, the result applies only to sets of truncated games with a fixed finite player set $N$ that are rich enough. If only traditional games $v$ on $\mathscr{F}$ and all elements of $X(N, v)$ have to be considered, then $\left(\mathbf{P}_{1}\right),\left(\mathbf{P}_{2}\right)$, and $\left(\mathbf{P}_{13}\right)$ are vacuously satisfied for any solution, and $\left(\mathbf{P}_{3}\right)$ is not sufficient to characterize a solution. However, the reduced game property may be regarded as a reduced game property w.r.t. the population (for more details, see the comment containing (6.5) of Maschler et al. [12]) for suitable reduced games (e.g., the Davis-Maschler reduced game). Nevertheless, for example, Sobolev's [24] traditional axiomatization of the prenucleolus requires an infinite universe of potential players. As soon as the domain is restricted to consist of all games with player sets belonging to a finite universe, Sobolev's [24] axioms (SIVA, anonymity, TCOV, SCOV, and the reduced game property w.r.t. Davis-Maschler reduced games), even together with the axiom requiring the solution to be contained in the least core $\left(\mathbf{P}_{3}\right)$, are not sufficient to characterize the prenucleolus, as can be seen by inspecting exercises 6.3.2 and 6.3.3 of Peleg and Sudhölter [18].

Third, theorem 5.2 of Kleppe et al. [10] axiomatizes a weighted prenucleolus exclusively for symmetric weight systems. Hence, Theorem 4 may be regarded as an advantage over the mentioned axiomatization because it also characterizes $p$-weighted (pre)nucleoli when $p$ is not symmetric.

Fourth, for the special class of nonsymmetric weight systems $p$ that result in $p$-weighted prenucleoli satisfying RSAM (see Section 3), we may proceed as in Section 4 and define, for any $z \in \mathbb{R}^{N}$ with $z \gg 0$ and $z(N)=n$, for any $v \in \Gamma^{b}$, and for any $\alpha \in \mathbb{R}$,

$$
v^{(\alpha, z, a d j)}(S)= \begin{cases}v(S), & \text { if } S \in \mathscr{F} \backslash \mathscr{E}(v), \\ v(S)-\alpha z(S), & \text { if } S \in \mathscr{E}(v) .\end{cases}
$$

Then we may say that a solution $\sigma$ on a set $\Gamma^{\prime}$ of games satisfies regular adjusted strong aggregate monotonicity $(R A S A M)$ w.r.t. $z$ if $\sigma\left(v^{(\alpha, z, a d j)}\right)+\{\alpha z\} \subseteq \sigma(v)$, whenever $\alpha>0$ and $v, v^{(\alpha, z, a d j)} \in \Gamma^{\prime} \cap \Gamma^{b}$.

Defining the weight system $p(z)=p$ by $p_{S}=\frac{1}{z(S)}$ for all $S \in \mathscr{\mathscr { F }}$, we receive, as in Section $5, v^{(\alpha)}=v_{(\alpha, p(z))}-\alpha z(\cdot)$ and $v^{(\alpha, z, a d j)}=v_{(\alpha, p(z), a d j)}-\alpha z(\cdot)$, respectively. Because RSAM and RASAM w.r.t. $z=\mathbb{1}^{N}$ coincide with ESD and EASD, respectively, the following result that is an immediate consequence of Theorem 4 generalizes Theorem 3 and Corollary 3.

Corollary 5. Let $z \in \mathbb{R}^{N}$ be such that $z \gg 0$ and $z(N)=n$. Let $p$ be the weight system defined by $p_{S}=\frac{1}{z(S)}$ for all $S \in \mathscr{F}$. Then we have the following:

(a) The p-weighted nucleolus is the unique solution on $\Gamma^{b}$ that satisfies SIVA, TCOV, SCOV, and RASAM w.r.t. z.

(b) On an arbitrary $\Gamma^{\prime}, \Gamma^{b} \subseteq \Gamma^{\prime} \subseteq \Gamma$, the p-weighted prenucleolus is the unique solution that satisfies SIVA, TCOV, $S C O V$, and RASAM and RSAM, both w.r.t. $z$. 


\section{Acknowledgments}

The authors are grateful to Bezalel Peleg for his valuable comments and to two anonymous referees of this journal for their constructive remarks that helped with the illustration of one of the main results and the discussion of the related relevant literature.

\section{References}

[1] Bondareva ON (1963) Some applications of linear programming methods to the theory of cooperative games [in Russian]. Problemi Kibernet 10:119-139.

[2] Calleja P, Llerena F (2017) Rationality, aggregate monotonicity and consistency in cooperative games: some (im)possibility results. Soc. Choice Welfare 48(1):197-220.

[3] Davis M, Maschler M (1965) The kernel of a cooperative game. Naval Res. Logist. Quart. 12(3):223-259.

[4] Davis M, Maschler M (1967) Existence of stable payoff configurations for cooperative games. Shubik M, ed. Essays in Mathematical Economics in Honor of Oskar Morgenstern (Princeton University Press, Princeton, NJ), 39-52.

[5] Derks JJM, Haller H (1999) Weighted nucleoli. Internat. J. Game Theory 28(2):173-187.

[6] Grotte JH (1970) Computation of and observations on the nucleolus, the normalized nucleolus and the central games. Unpublished master's thesis, Cornell University, Ithaca, New York.

[7] Hokari T (2000) The nucleolus is not aggregate-monotonic on the domain of convex games. Internat. J. Game Theory 29(1):133-137.

[8] Housman D, Clark L (1998) Core and monotonic allocation methods. Internat. J. Game Theory 27(4):611-616.

[9] Justman M (1977) Iterative processes with "nucleolar" restrictions. Internat. J. Game Theory 6(4):189-212.

[10] Kleppe J, Reijnierse H, Sudhölter P (2016) Axiomatizations of symmetrically weighted solutions. Ann. Oper. Res. 243(1-2):37-53.

[11] Kohlberg E (1971) On the nucleolus of a characteristic function game. SIAM J. Appl. Math. 20(1):62-66.

[12] Maschler M, Potters JAM, Tijs SH (1992) The general nucleolus and the reduced game property. Internat. J. Game Theory 21(1):85-105.

[13] Megiddo N (1974) On the monotonicity of the bargaining set, the kernel, and the nucleolus of a game. SIAM J. Appl. Math. 27(2):355-358.

[14] Orshan G (1993) The prenucleolus and the reduced game property: Equal treatment replaces anonymity. Internat. J. Game Theory 22(3): 241-248.

[15] Orshan G, Sudhölter P (2003) Reconfirming the prenucleolus. Math. Oper. Res. 28(2):283-293.

[16] Oswald J, Derks J, Peters H (1998) Prenucleolus and nucleolus of a cooperative game: Characterizations by tight coalitions. Aportaciones Matematicas: Comunicaciones 24:197-216.

[17] Peleg B (1986) On the reduced game property and its converse. Internat. J. Game Theory. 15(3):187-200.

[18] Peleg B, Sudhölter P (2007) Introduction to the Theory of Cooperative Games, 2nd ed. Theory and Decision Library, Series C: Game Theory, Mathematical Programming and Operations Research, vol. 34 (Springer, Berlin)

[19] Schmeidler D (1969) The nucleolus of a characteristic function game. SIAM J. Appl. Math. 17(6):1163-1170.

[20] Schmeidler D (1972) Cores of exact games. J. Math. Anal. Appl. 40:214-225.

[21] Shapley LS (1953) A value for n-person games. Kuhn HW, Tucker AW, eds. Contribution to the Theory of Games II. Annals of Mathematics Studies, vol. 28 (Princeton University Press, Princeton, NJ), 307-317.

[22] Shapley LS (1967) On balanced sets and cores. Naval Res. Logist. Quart. 14(3):453-460.

[23] Shapley LS (1971) Cores of convex games. Internat. J. Game Theory 1:11-26.

[24] Sobolev AI (1975) The characterization of optimality principles in cooperative games by functional equations. Vorobiev NN, ed. Mathematical Methods in the Social Sciences, vol. 6 [in Russian] (Lithuanian Academy of Sciences, Vilnius, Lithuania), 95-151.

[25] Sudhölter P (1997) The modified nucleolus: Properties and axiomatizations. Internat. J. Game Theory 26(2):147-182. 